\title{
Programa de Aulas Limpias y Educación ambiental en estudiantes de primero de secundaria I.E Miraflores - Lima 2019
}

\section{Program of Clean Classrooms and Environmental Education in students of first year of high school I.E Miraflores - Lima 2019}

\author{
Madeleine Ludeña Pelayes ${ }^{1}$, Abelardo Rodolfo Campana Concha ${ }^{2}$
}

\begin{abstract}
RESUMEN
Objetivo: Determinar la influencia del Programa de Aulas Limpias frente a la Educación ambiental en estudiantes de primero de secundaria I.E Miraflores-Lima 2019. Materiales y Métodos: Es un estudio cuasi - experimental con una muestra de 18 estudiantes, a quienes se les aplicó un pre y post test. Se utilizó el estadístico SPSS. Resultados: Los datos estadísticos nos permiten afirmar que las medias logradas por el grupo de estudio, respecto a la evaluación inicial aplicada a los estudiantes refleja un promedio de 11,00, luego de la aplicación del Programa Aulas Limpias, el post test arrojó como resultado una media de 18,00 puntos. De modo que el aprendizaje mediante esta modalidad resultó positivo porque se incrementó en 7,00 puntos. Por lo cual se acepta la hipótesis de trabajo. Confirmándose que este programa influenció favorablemente en la educación ambiental. Conclusión: La aplicación del programa Aulas Limpias mejoró significativamente en la educación ambiental en estudiantes del primer grado de secundaria en la institución estudiada, respecto al aprendizaje de situaciones ambientales, la actitud ambiental y sobre todo del conocimiento ambiental.
\end{abstract}

Palabras clave: Aulas Limpias, Educación ambiental, situación ambiental, actitud ambiental, conocimiento ambiental.

\section{ABSTRACT}

Objective: To determine the influence of the Clean Classroom Program against environmental Education in IE Miraflores-Lima 2019 high school students. Materials and Methods: It is a quasi-experimental study with a sample of 18 students, to whom a pre and post test The SPSS statistic was used. Results: The statistical data allow us to affirm that the means achieved by the study group, with respect to the initial evaluation applied to the students, reflects an average of 11,00, after the application of the Clean Classrooms Program, the post test resulted in an average of 18,00 points. So learning through this modality was positive because it increased by 7,00 points. Therefore, the working hypothesis is accepted. Confirming that this program favorably influenced environmental education. Conclusion: The application of the Clean Classrooms program improved significantly in environmental education in students of the first grade of secondary school in the institution studied, regarding the learning of environmental situations, the environmental attitude and especially of environmental knowledge.

Keywords: Clean Classrooms, Environmental education, environmental situation, environmental attitude, environmental knowledge.

\section{INTRODUCCIÓN}

En la actualidad muy poco se trabaja en la educación ambiental. El sector Educación no es ajeno a este tema y reconoce que el liderazgo que se ejerza en las Instituciones Educativas es imprescindible para alcanzar resultados evidentes que demuestren la calidad y la eficiencia en el servicio educativo.

En las Instituciones Educativas donde exista educación ambiental tanto alumnos como docente se evidencia procesos de cambio ya que es capaz de conducir hacia objetivos y metas que permitan mejorarla condición de vida en cuanto al cuidado del medio ambiente.

La educación ambiental que se ejerza en las instituciones educativas ya sea educación afectiva, activa, cognitiva y conativa deben buscar el comprometer a las personas con el trabajo en equipo, desarrollar un mejor discernimiento que le permita trascender y ser significativa para la comunidad.

Nuestro país ha demostrado durante muchos años una falta de educación ambiental: las personas abren las ventanas de los micros para arrojar la basura, quema de basura en algunas zonas de la capital, falta de recojo de basura por las calles, mercados sucios, basura en los alrededores de algunas escuelas, falta de limpieza en los mercados, playas sucias.

Últimamente En el Perú se han difundido ideas, sucesos, normas, iniciativas empresariales y políticas gubernamentales referidas al ambiente. Existe un mayor conocimiento y una buena disposición hacia lo que significa un mayor cuidado de nuestros paisajes, recursos naturales y ecosistemas, pero eso no compromete a acciones decididas y convencidas, ya que aún no existe una cultura de educación ambiental, es necesario una iniciativa fuerte desde la escuela y las municipalidades que involucre a los hogares y a toda la comunidad, de tal manera que ya no se vean calles, aulas sucias y ambientes sucios.

De alguna manera ya se ha comenzado a dar más énfasis a la educación ambiental con asuntos como, por ejemplo: la declaración del día mundial del medio ambiente, creación del ministerio de ambiente. En estos últimos años se ha observado diversas movilizaciones, pero enfocadas al cuidado de los recursos naturales en donde se ven amenazados especies o en donde se ve afectada una población determinada; sin embargo aún no se ve un compromiso de educación ambiental en donde se vea involucrada la conducta de las personas hacia una educación ambiental.

Cada uno de nosotros somos parte del medio que nos rodea, vivimos, nos nutrimos y nos servimos de él, sin embargo, en nuestro colegio somos testigos de la falta de educación ambiental que vivimos desde nuestras aulas: papeles en el piso, patios sucios después del refrigerio, falta del cuidado de los ambientes de la institución (baños), existe falta de educación ambiental desde los profesores que dictan las clases, dado que algunos de ellos realizan las clases en situaciones no limpias, no somos ajenos al cuidado de nuestro planeta y es necesario asumir nuestra responsabilidad y compromiso con el cuidado y conservación de nuestro planeta, por 
esta razón vemos la necesidad de hacer un programa que genere educación ambiental en cada uno de los estudiantes y estos a su vez puedan hacer efecto multiplicador en sus hogares.

Algunos estudios nos permiten conocer un poco de la realidad de las variables analizadas. Por ejemplo, Valdez (2013) evaluó el proceso de incorporación de la educación ambiental en dos escuelas secundarias de Saltillo, Coahuila, teniendo en cuenta las representaciones sociales de los estudiantes y educadores sobre el medio ambiente, problemática ambiental, sustentabilidad y transversalidad; a fin de plantear pautas que reorienten dicho proceso de incorporación de la educación ambiental en este nivel educativo, concluyendo finalmente en emplear en el quehacer cotidiano de las instituciones educativas la educación ambiental con sus principales características de interdisciplinariedad y complejidad a través de la colaboración de los diversos actores del proceso educativo de toda la institución.

En el ámbito nacional tenemos a Estrada (2017), quien determinó la relación entre la Educación ambiental y conservación del medio ambiente en los alumnos del cuarto grado de primaria de la I.E. 6069 UGEL 01 Villa el Salvador. Lima, 2016, llegando a la conclusión que existen evidencias para afirmar que la Educación ambiental se relaciona significativamente con la conservación del medio ambiente.

El presente trabajo de investigación pretende determinar la influencia del Programa de Aulas Limpias frente a la Educación ambiental en estudiantes de primero de secundaria I.E Miraflores-Lima 2019, del cual se busca que el programa mejore sus conocimientos con respecto al medio ambiente.

\section{MÉTODOS YMATERIALES}

La investigación corresponde a un estudio cuasi experimental teniendo una muestra de 18 estudiantes del primero de secundaria I.E Miraflores-Lima 2019. Se aplicó un pre y post test, los datos fueron proporcionados en el programa SPSS.

\section{RESULTADOS}

\section{Prueba de hipótesis general}

HG. El Programa Aulas Limpias influye en la educación ambiental en estudiantes de primero de secundaria I.E Miraflores-Lima 2019

Tabla 1

Hipótesis general

\begin{tabular}{lcccc}
\hline & N & Media & $\begin{array}{c}\text { Desviación } \\
\text { típ. }\end{array}$ & $\begin{array}{c}\text { Error típ. de la } \\
\text { media }\end{array}$ \\
\hline Pre test & 18 & 11,00 & 2,315 & 0,454 \\
Pos test & 18 & 18,00 & 3,807 & 0,747 \\
\hline
\end{tabular}

\section{Contrastación de la hipótesis específica 1}

H1. El Programa Aulas Limpias influye en situaciones ambientales en estudiantes de primero de secundaria I.E Miraflores-Lima 2019.
Tabla 2

Hipótesis especifica 1

\begin{tabular}{lcccc}
\hline \multicolumn{5}{c}{ Estadísticos para una muestra } \\
\hline & $\mathrm{N}$ & Media & $\begin{array}{c}\text { Desviación } \\
\text { típ. }\end{array}$ & $\begin{array}{c}\text { Error típ. de } \\
\text { la media }\end{array}$ \\
\hline Pre test & 26 & 13,00 & 3,514 & 0,689 \\
Pos test & 26 & 17,00 & 3,349 & 0,657 \\
\hline
\end{tabular}

Contrastación de la hipótesis específica 2

H2. El Programa Aulas Limpias influye en actitud ambiental cognitiva en estudiantes de primero de secundaria I.E Miraflores-Lima 2019

Tabla 3

Hipótesis específica 2

\section{Estadísticos para una muestra}

\begin{tabular}{lcccc}
\hline & $\mathrm{N}$ & Media & $\begin{array}{c}\text { Desviación } \\
\text { típ. }\end{array}$ & $\begin{array}{c}\text { Error típ. de la } \\
\text { media }\end{array}$ \\
\hline Pre test & 26 & 12,00 & 4,135 & 0,811 \\
Pos test & 26 & 16,00 & 4,450 & 0,873 \\
\hline
\end{tabular}

\section{Contrastación de la hipótesis específica 3}

H3. El Programa Aulas Limpias influye en conocimiento ambiental en estudiantes de primero de secundaria I.E Miraflores-Lima 2019

Tabla 4

Hipótesis especifica 3

$$
\text { Estadísticos para una muestra }
$$

\begin{tabular}{lcccc}
\hline & $\mathrm{N}$ & Media & $\begin{array}{c}\text { Desviación } \\
\text { típ. }\end{array}$ & $\begin{array}{c}\text { Error típ. de la } \\
\text { media }\end{array}$ \\
\hline Pre test & 26 & 14,00 & 5,281 & 1,036 \\
Pos test & 26 & 18,00 & 4,662 & 0,914 \\
\hline
\end{tabular}

\section{DISCUSIÓN}

En base a los análisis realizados en la hipótesis general, se determinó que las medias logradas por el grupo de estudio, respecto a la evaluación inicial aplicada a los estudiantes refleja un promedio de 11,00 , luego de la aplicación del Programa Aulas Limpias, el post test arrojó como resultado una media de 18,00 puntos. De modo que el aprendizaje mediante esta modalidad resultó positivo porque se incrementó en 7,00 puntos. Por lo cual se acepta la hipótesis de trabajo. Confirmándose que este programa influenció favorablemente en la educación ambiental.

En el análisis de la primera hipótesis específica, nos permitió confirmar que las medias logradas por el grupo de estudio, respecto a la evaluación inicial aplicada a los estudiantes arrojó como resultado un promedio de 13,00; luego de la aplicación del Programa Aulas Limpias, se presentó el post test con una media de 17,00 puntos. De modo que el aprendizaje mediante esta modalidad resultó positivo porque se incrementó en 4,00 puntos. Por 
lo cual se acepta la hipótesis de trabajo, con lo cual este programa influenció favorablemente las situaciones ambientales de los estudiantes.

En cuanto al análisis de la segunda hipótesis específica se demostró que las medias logradas por el grupo de estudio, respecto a la evaluación inicial aplicada a los estudiantes arrojó como resultado un promedio de 12,00;, luego de la aplicación del Programa Aulas Limpias, se presentó el post test con una media de 16,00 puntos. De modo que el aprendizaje mediante esta modalidad resultó positivo porque se incrementó en 4,00 puntos. Por lo cual se acepta la hipótesis de trabajo. Se confirmó que este programa influenció favorablemente la actitud ambiental cognitiva de los estudiantes.

En la tercera hipótesis específica, se encontró que las medias logradas por el grupo de estudio, respecto a la evaluación inicial aplicada a los estudiantes arrojó como resultado un promedio de 14,00; luego de la aplicación del Programa Aulas Limpias, se presentó el post test con una media de 18,00 puntos. De modo que el aprendizaje mediante esta modalidad resultó positivo porque se incrementó en 4,00 puntos. Por lo cual se acepta la hipótesis de trabajo. Con lo cual se confirmó que este programa influenció favorablemente en el conocimiento ambiental de los estudiantes.

Velázquez (2017), en su investigación sobre educación ambiental en estudiantes identificó que solo se evidencia en algunas asignaturas mediante el desarrollo de actividades que generan cultura y una conciencia ambiental, a pesar de haber tenido resultado alentador con el comportamiento de los estudiantes no son suficientes para desarrollar una buena educación ambiental en la institución, situación que también hemos comprobado se notó el incremento en los conocimientos ambientales pero este tiene que ser alimentado constantemente.

A diferencia de lo encontrado por Egaña (2018), su estudio solo se limitó a describir la problemática de la educación ambiental en las escuelas de Chile, que en resumen son básicas y no se ahondan mucho, por lo cual alerta urgentemente usar didácticas que le permitan a los estudiantes hacer una conexión rápida entre realidad ambiental y sus historias de vida para agilizar el proceso de aprendizaje significativo, aunque no lo comprobó con un programa nuestros resultados comprueban que es necesario implementarlos para ir formando en los estudiantes conciencia ambiental.

\section{CONCLUSIONES}

En base a los análisis realizados encontramos lo siguiente:

La aplicación del programa Aulas Limpias mejoró significativamente en la educación ambiental en estudiantes del primer grado de secundaria en la institución estudiada, respecto al aprendizaje de situaciones ambientales, la actitud ambiental y sobre todo del conocimiento ambiental.

Asimismo el Programa Aulas Limpias mejoró significativamente en situaciones ambientales: como el cuidado del medio ambiente, explicar porque suceden los cambios ambientales y describen sobre el ambiente saludable.

A través del empleo de programa de Aulas Limpias se mejoró notablemente, respecto a la actitud ambiental: participan en el cuidado, solución, valoración y la selección de materiales.
Mediante el programa de Aulas Limpias los estudiantes mejoraron: frente a las formas de actuar, conocen las normas de seguridad, sobre el impacto ambiental.

\section{AGRADECIMIENTOS}

Agradecimiento a todos los que formaron parte de este estudio

\section{REFERENCIAS BIBLIOGRÁFICAS}

Egaña, M. (2018). Significado otorgado a la Educación Ambiental por diversos tipos de establecimientos educacionales en Chile: Un análisis de experiencias publicadas. (Tesis Pre grado) Universidad de Chile, $\mathrm{C} \mathrm{h} \mathrm{i} \mathrm{l} \mathrm{e.} \mathrm{R} \mathrm{e} \mathrm{c} \mathrm{u} \mathrm{p} \mathrm{e} \mathrm{r} \mathrm{a} \mathrm{d} \mathrm{o} \mathrm{e} n$ : http://repositorio.uchile.cl/bitstream/handle/2250/14 7358/ega\%c3\%b1a\%20pacheco.pdf?sequence=1 \&isAllowed =y

Estrada, R. (2017). Educación ambiental y conservación del medio ambiente en los alumnos del cuarto grado de primaria de la I.E. 6069 UGEL 01 de Villa el Salvador. Lima. 2016 (Tesis Posgrado) UCV, Lima, Perú.

Valdez, R. (2013). Educación ambiental en la escuela secundaria pública: una evaluación desde la teoría de las representaciones sociales en un caso de estudio en Saltillo, Coahuila (Tesis Posgrado). Universidad Autónoma De Nuevo León, México. $R$ e c u p e r a d o e $n$ : http://cdigital.dgb.uanl.mx/te/1080253512.PDF

Velásquez, Y. (2017). La educación ambiental, una reflexión en torno a la relación entre comunidad educativa y medio ambiente, desde los imaginarios colectivos y espacios de la institución educativa playa rica, en el municipio del Tambo-Cauca (Tesis Posgrado). Universidad de Manizales, Colombia. $\mathrm{R}$ e c u p e r a d o e $n$ : http://ridum.umanizales.edu.co:8080/xmlui/bitstrea $\mathrm{m} /$ handle/6789/3149/Tesis\%20Yenni\%20velasquez .pdf? sequence $=1$ \&isAllowed $=y$ 